\title{
Erratum to: Influence of diffusion on the stability of equilibria in a reaction-diffusion system modeling cholera dynamic
}

\author{
Florinda Capone ${ }^{1}$. Valentina De Cataldis ${ }^{1}$. \\ Roberta De Luca ${ }^{1}$
}

Published online: 22 July 2015

(C) Springer-Verlag Berlin Heidelberg 2015

\section{Erratum to: J. Math. Biol. DOI 10.1007/s00285-014-0849-9}

In a private communication, Zengji Du and Rui Peng (Jiangsu Normal University) pointed out that, in the originally published article, when performing the stability analysis of the disease free equilibrium (DFE) we use a wrong argument, the following: $\int_{\Omega} \varphi d \Omega=0$ (null average condition). Indeed, in this case, the perturbations to infective, bacteria and removed population are (essentially) positive and hence the null average condition is biologically meaningless. Avoiding the null average condition, under the Neumann boundary conditions, the stability threshold for DFE is the same as that one obtained from the corresponding ODE system, since-in this case- the lowest eigenvalue of the spectral problem $\{\Delta \varphi=\alpha \varphi$, in $\Omega ; \nabla \varphi \cdot \mathbf{n}=0$, on $\partial \Omega\}$ is $\bar{\alpha}=0$ and hence Theorem 2 and Theorem 4 (the latter only for DFE) continue to hold with $R_{0}^{*}=1$ (cfr. (26)).

However, we remark that $R_{0}^{*}>1$ holds if -at least in a small part of the boundary with positive measure-one requires conditions different from the Neumann ones. Thus, if to (1) the mixed boundary conditions are appended (Capone et al. 2013), i.e.:

The online version of the original article can be found under doi:10.1007/s00285-014-0849-9.

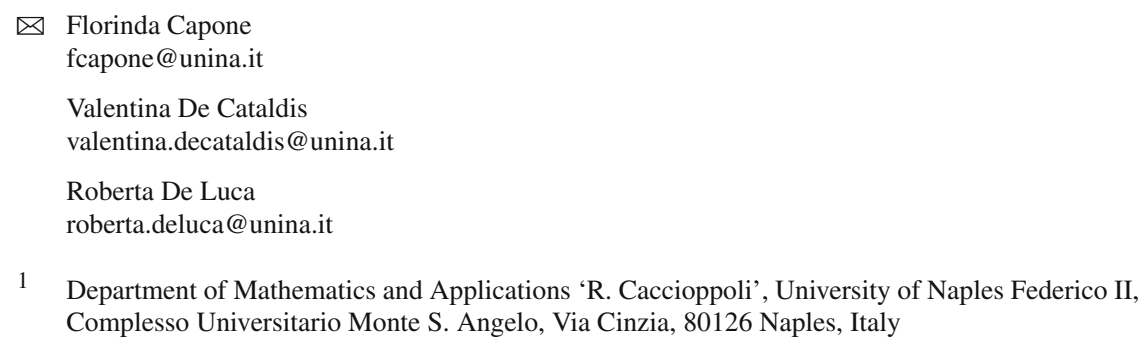

1 Department of Mathematics and Applications 'R. Caccioppoli', University of Naples Federico II, Complesso Universitario Monte S. Angelo, Via Cinzia, 80126 Naples, Italy 


$$
\begin{cases}S=\bar{S}, I=\bar{I}, B=\bar{B}, R=\bar{R}, & \text { on } \Sigma_{1} \times \mathbb{R}^{+} \\ \nabla S \cdot \mathbf{n}=0, \nabla I \cdot \mathbf{n}=0, \nabla B \cdot \mathbf{n}=0, \nabla R \cdot \mathbf{n}=0, & \text { on } \Sigma_{2} \times \mathbb{R}^{+},\end{cases}
$$

where $\partial \Omega=\Sigma_{1} \cup \Sigma_{2}, \Sigma_{1} \cap \Sigma_{2}=\emptyset, \Sigma_{1} \neq \emptyset$, meas $\left(\Sigma_{1}>0\right)$, $\mathbf{n}$ being unit outward normal on $\Sigma_{2}$ and $\bar{S}, \bar{I}, \bar{B}, \bar{R}$ being non negative constants, all the mathematical results from Section 3 onward of our original paper hold true, with $\bar{\alpha}(\Omega)>0$ given by (14) and $W^{*}(\Omega)$ replaced by

$\tilde{W}(\Omega)=\left\{\varphi \in W^{1,2}(\Omega) \cap W^{1,2}(\partial \Omega): \varphi=0\right.$, on $\Sigma_{1} \times \mathbb{R}^{+}, \nabla \varphi \cdot \mathbf{n}=0$, on $\left.\Sigma_{2} \times \mathbb{R}^{+}\right\}$.

The following typos and inserts for clarification in the original paper should be noticed:

(i) in formula (6) should be $\tilde{N}_{0}$ rather than $N_{0}$, where $\tilde{N}_{0}$ is a positive constant. Precisely the inequality (6) can be obtained following, step by step, the procedure in Capone et al. (2013) which is similar to the procedure used to prove Theorem 1 in the original paper. $N_{0}$ has to be replaced by $\tilde{N}_{0}$ also in the statement and proof of Theorem 1 and in the definition of $c_{i}, \forall i \in\{1,2,3,4,5,6\}, \bar{c}$;

(ii) in formula (10), $\bar{B}$ has to be deleted;

(iii) Remark 2 is not necessary and hence it has to be deleted;

(iv) in the statement and proof of Lemma 4 and in the definition of $m$ in Theorem 4, $\mu_{3}$ and $U_{3}$ have to be replaced by $\mu_{2}$ and $U_{2}$, respectively. 